\title{
Alle origini del romanzo epistolare: la risposta come matrice narrativa elementare
}

\section{Magda Campanini Catani}

\section{(2) OpenEdition}

1 Journals

\section{Edizione digitale}

URL: https://journals.openedition.org/studifrancesi/37023

DOI: $10.4000 /$ studifrancesi.37023

ISSN: 2421-5856

\section{Editore}

Rosenberg \& Sellier

\section{Edizione cartacea}

Data di pubblicazione: 15 décembre 2004

Paginazione: 441-456

ISSN: 0039-2944

\section{Notizia bibliografica digitale}

Magda Campanini Catani, «Alle origini del romanzo epistolare: la risposta come matrice narrativa elementare», Studi Francesi [Online], 144 (XLVIII | III) | 2004, online dal 30 novembre 2015, consultato il 08 mai 2021. URL: http://journals.openedition.org/studifrancesi/37023 ; DOI: https://doi.org/10.4000/ studifrancesi.37023

\section{(c) (i) (9)}

Studi Francesi è distribuita con Licenza Creative Commons Attribuzione - Non commerciale - Non opere derivate 4.0 Internazionale. 


\section{Alle origini del romanzo epistolare: la risposta come matrice narrativa elementare}

Ecrire une lettre et la faire jeter à la poste; recevoir la réponse, la lire et la brûler, voilà la correspondance réduite à sa plus simple expression. ${ }^{1}$

Nello studio delle origini del romanzo epistolare europeo, e francese in particolare, è stato a più riprese sottolineato dalla critica il ruolo non secondario svolto dai secrétaires nel processo di affermazione del genere. È infatti ormai riconosciuto il legame di continuità tra manuali di epistolografia e aggregazioni di lettere dall'impianto più specificamente narrativo ${ }^{2}$, che precorrono lo sviluppo autonomo del roman par lettres. Proseguendo e approfondendo questa direzione di ricerca, indirizzerò la mia indagine nell'ambito dei formulari e dei florilegi epistolari pubblicati in Francia tra Cinquecento e Seicento. La scelta del corpus testuale di riferimento terrà conto di alcune opere particolarmente rappresentative delle diverse tendenze della produzione del tempo che, a partire dalla prima metà del Cinquecento, conosce una grande espansione indirizzata soprattutto al versante pedagogico. Conformemente al grande esempio di Erasmo e degli epistolografi italiani, la lettera, prima latina e poi anche volgare, si afferma come importante strumento di educazione e affida spesso a manuali la redazione di precetti e modelli di scrittura adattabili alle più svariate circostanze. Nei formulari di questo tipo si moltiplica infatti il numero delle tipologie epistolari illustrate, che, simulando delle micro-situazioni comunicative rigidamente

(1) Balzac, La physiologie du mariage. Ed. présentée par Maurice Regard, Paris, Garnier, 1968, p. 213.

(2) Sulla problematica della finzione narrativa in opere epistolari di impianto manualistico tra Cinquecento e Seicento si vedano in particolare $\mathrm{i}$ seguenti contributi critici: A. PIZzorusso, La poetica del romanzo in Francia, Caltanissetta-Roma, S. Sciascia Editore, 1962, pp. 79-98; B. BRAY, L'art de la lettre amoureuse. Des manuels aux romans, $\mathrm{La}$ Haye-Paris, Mouton, 1967; A. Pizzorusso, Boursault et le roman par lettres, in «Revue d'Histoire littéraire de la France», maggio-agosto 1969, pp. 525-539; R. DuchÊNE, Réalité vécue et réussite littéraire: le statue particulier de la lettre, in «Revue d'Histoire littéraire de la France», marzo-aprile 1971, pp. 177-193; L. VERSINI, Le roman épistolaire, Paris, P.U.F., 1979, cap. II; S. L. CARrEL, Le soliloque de la passion féminine ou le dialogue illusoire: étude d'une formule monophonique de la littérature épistolaire, Paris- Tübingen, Place-Narr, 1982; J. Altman, Epistolarity, approaches to a form, Ohio State University Press, 1982; J. Altman, The letter bookas literary institution 1539-1789: toward a cultural bistory of published correspondances in France, in «Yale French Studies», 71, 1986, pp. 17-62; J. Chupeau, Puget de la Serre et l'esthétique épistolaire: les avatars du «Secrétaire de la Cour», in «Cahiers de l'Association Internationale des Etudes Françaises», maggio 1987, pp. 111-218; G. MALQUORI FONDI, Introduction à François d'Aubignac, Le Roman des lettres, Paris-Seattle-Tübingen,
«Papers on French 17th Century literature», 1989 pp. XIX-XCVI; G. MalQuORI FONDI, Conversations d'amour par lettres: un récueil méconnu de Le Pays, un roman inconnu de Pradon, in Art de la lettre, art de la conversation à l'époque classique en France, Actes du Colloque de Wolfenbüttel, 1991, publiés par B. Bray et C. Strosetzki, Paris, Klincksieck, 1995, pp. 257-270; G. MALQUORI FONDI, «Vorrei e non vorrei», ou les lettres d'amour de la jeune Iris à Timandre, in Correspondances. Mélanges offerts à R. Duchêne, Tübingen, Narr, 1992, pp. 505-512; F. PIVA, Le "Commerce Galant": essai de définition, in C. BERNARD - J. PRADON, Le Commerce Galant. Texte établi, présenté et annoté par F. PIVA, Fasano-Paris, Schema-Nizet, 1996, pp. 11-82. B. BRAY, Le roman par lettres ou la fiction dénoncée, in Perspectives de la recherche sur le genre narratif français du XVIIe siècle, Pisa-Genève, ETS-Slatkine, 2000, pp. 187-211. Più in generale, sul rapporto ambivalente tra veridicità e finzione ai margini della narrazione romanzesca si rinvia a S, YAHALOM, Du non-littéraire au littéraire. Sur l'élaboration d'un modèle romanesque au XVIIIe siècle, in «Poétique», 44 1980, pp. 406-421; H. BOYER, La communication épistolaire comme stratégie romanesque, in «Semiotica», 39, 1982, pp. 21-44; J. HERMAN, Le mensonge romanesque, Amsterdam-Leuven, Rodopi-Leuven University Press, 1989; G. LANE-MERCIER, La parole romanesque, Paris-Ottawa, Klincksieck-Presses de l'Université d'Ottawa, 1989; D. CoHN, Le propre de la fiction, Paris, Seuil, 2001. 
codificate, spaziano dalle lettere di complimento a quelle di ringraziamento, d'amore, di raccomandazione, di scuse o di condoglianze.

La crescente diffusione di "segretari" epistolari che conoscono, soprattutto nel Seicento, un notevole successo editoriale, affonda le radici in una tradizione normativa rigidamente strutturata che si consolida nel corso del Cinquecento coniugando i rigidi canoni delle artes dictaminis medievali con le innovazioni di matrice erasmia$\mathrm{na}^{3}$. Nel lungo e graduale processo di affrancamento dall'impostazione medievale si assiste ad una continua ripresa e rielaborazione di norme retoriche, stilemi e materiali epistolari che si intersecano nell'elaborazione dei diversi trattati. Ripercorrendo solo a grandissime linee l'evoluzione della retorica epistolare francese cinquecentesca, si può notare come nel corso della prima metà del secolo l'epistolografia risenta fortemente dell'influsso di Pierre Fabri che, nella sezione del suo Grant et Vray Art de pleine rhetorique (1521) dedicata alla composizione delle lettere, ripropone le ripartizioni retoriche medievali rifacendosi soprattutto alla Summa dictaminis di Guido Faba (1228-1229 ca.). Ne deriva in particolar modo il canone tripartito, vero e proprio cardine della sua teoria epistolare ${ }^{4}$ e principio ispiratore dell'ampio inventario di Lettres missives che completano il trattato con la funzione di modelli. Avanzando nel secolo, con la consapevolezza di tratteggiare in maniera del tutto incompleta gli sviluppi della teorizzazione epistolare, assistiamo ad un allontanamento progressivo dal Grant et Vray Art di Fabri. È questa una tendenza evolutiva a cui non risulta certamente estranea l'influenza del De conscribendis epistolis erasmiano, pietra angolare per il rinnovamento dell'epistolografia, opera che contrappone al formalismo delle artes dictandi di stampo medievale l'apertura verso le infinite possibili sfumature dello stile epistolare, «versipellis ac polypus» ${ }^{5}$. Il nostro campo d'indagine si limiterà tuttavia alla produzione in francese, al cui interno spicca la rielaborazione del trattato di Fabri che Pierre Durand dà alle stampe nel 1553. Si tratta dello Stile et maniere de composer, dicter et escrire toutes sortes d'Epistres ou lettres missives, tant par responce qu'autrement ${ }^{6}$, compilazione di ampio respiro che costituisce una sorta di summa della trattatistica epistolare in lingua volgare dell'epoca. Rispetto alle elaborazioni teoriche precedenti, lo Stile et maniere testimonia una minor rigidità nell'applicazione delle norme retoriche in favore di una maggior attenzione allo "stile", nozione che segue meno da vicino le rigide articolazioni dell' elocutio e induce di conseguenza

(3) Sulla retorica epistolare medievale e sul valore profondamente innovativo del De conscribendis epistolis di Erasmo si veda in particolare E. R. CURTIUS, La littérature européenne et le Moyen-Age latin, Paris, P.U.F., 1956, cap. IV, pp. 142-144; J.J. MuRPHY, Rhetorics in the Middle Ages, a History of Rhetorical Theory from St. Augustine to the Renaissance, Berkeley-Los Angeles-London, University of California Press, 1974; M. Fumaroli, Genèse de l'épistolographie classique: rhétorique bumaniste de la lettre de Pétrarque à Juste Lipse, in «Revue d'Histoire littéraire de la France», 78, 1987, pp. 886-900; J.R. HENDERSEN, Erasmus on the Art of Letter-Writing, in Renaissance Eloquence. Studies in the Theory and Practice of Renaisssance Rhetoric, Edited by J. J. Murphy, Berkeley-Los Angeles-London, University of California Press, 1983, pp. 331335; TH. O. BEEBEE, Epistolary Fiction in Europe (1500-1850), Cambridge, Cambridge University Press, 1999 , cap. 2.

(4) «Toute epistre est partie en trois, comme un argument qui est de maieur, mineur et de conclusion, que les orateurs disent la cause, l'intention et la consequence» (P. FABRI, Le grand et vrai art de pleine rbétorique. Publié avec introduction, notes et glossaire par A. Héron, Genève, Slatkine Reprints, 1969, I, p. 199).

La sezione epistolare del trattato di Fabri verrà pubblicata autonomamente nel Prothocolle des Secretaires et aultres gens desirans sçavoir l'art et maniere de dicter en bon françoys toutes lettres missives et epistres en prose, Lyon, Amoulet, 1534.

Sui legami del trattato di Fabri con l'epistolografia medievale cfr. in particolare CL. LA CHARITÉ, Le stile et maniere de composer, dicter, et escrire toutes sortes d'epistres, ou lettres missives (1553). De la Dispositio tripartite de Pierre Fabri au poulpe épistolaire d'Erasme, in L'epistolaire au XVIe siècle, «Cahiers V. L. Saulnier», 18, Paris, Editions Rue d'Ulm-Presses de l'Ecole Normale Supérieure, 2001, pp. 17-30.

(5) Opera Omnia Desideri Erasmi Roterodami, Amsterdam, North-Holland Publishing Company, 1971, I, 2, p. 223.

(6) Lyon, Jean Temporal, 1553. 
ad una certa flessibilità del discorso epistolare, così come lo confermano i numerosi esempi di lettere che arricchiscono il manuale e nelle quali la strutturazione tripartita non è più esplicitamente segnalata. Le qualità innovative del manuale di Durand non nascondono tuttavia palesi emprunts ricavati non solo da Fabri, ma anche da un altro formulario sul quale ci soffermeremo più avanti, la Maniere d'escrire par responce di Jean Quinerit de Mousne. Come usualmente avviene all'epoca, anche lo Stile et maniere, che peraltro conosce una considerevole fortuna, verrà a sua volta ripreso da altri autori, contribuendo così ad alimentare il tessuto composito e stratificato della trattatistica epistolare rinascimentale, ove costantemente si incrociano echi di opere diverse e si rilevano riprese anche letterali di intere porzioni di testi.

All'interno di questo panorama teorico così variegato, che avremo comunque modo di precisare più dettagliatamente, ci soffermeremo ora sulla tipologia epistolare oggetto del nostro interesse: la lettera di risposta così come si configura nella corrispondenza d'amore e nelle lettere familiari.

Tra le numerose relazioni interpersonali proposte come modelli di comunicazione scritta, la relazione amorosa gode, nel periodo preso in esame, di un'attenzione crescente da parte degli epistolografi che spesso le dedicano intere sezioni delle loro opere se non addirittura l'intero volume. Nella fortuna registrata dai formulari galanti a partire dalla metà del Cinquecento convergono sicuramente almeno due fattori: da un lato la tradizione, mai venuta meno, delle Heroides ovidiane, frequentemente tradotte e a volte rielaborate o riproposte in veste autonoma, dall'altro la grande influenza delle composizioni epistolari italiane e spagnole, amplificata da un gran numero di traduzioni in francese ${ }^{8}$. In questo contesto le lettere d'amore, nobilitate dagli antecedenti latini e volgari, tendono ad assumere una dimensione indipendente rispetto alle altre categorie di lettere accolte nei manuali, aggregandosi talvolta in appendici autonome non di rado distinte da un sottotitolo.

Nelle opere di più solido impianto teorico, come il Grant et Vray Art de pleine Rhetorique, si ritrovano precise indicazioni sulla composizione di questo genere di missive, che devono risultare sempre rigidamente tripartite, sia che si tratti di lettere in cui si dichiari all'interlocutore «l'amour vertueuse de quoy on l'ayme», sia che si voglia «rescripre d'amour vitieuse à quelque belle ieune fille pour lui desclarer son amour» ${ }^{9}$. Anche nelle lettere d'amore si raccomanda dunque una rigorosa scansione dei contenuti, la cui disposizione resta legata a precisi canoni retorici. Pur con un progressivo allentamento della rigidità articolatoria rilevabile nel corso dei decenni, si riconosce infatti nella maggior parte della produzione manualistico-antologica una matrice convenzionale che presiede all'organizzazione dei materiali presentati.

Tuttavia, nonostante la forte impronta stereotipata, la lettre amoureuse inserita nelle raccolte o nell'insieme di modelli che solitamente integrano i manuali tende a costituirsi come luogo privilegiato della deriva narrativa, come spazio testuale

(7) Lyon, J. Moderne, s.d. [1548].

(8) Si ricordano tra tutti l'Historia de duobus amantibus (1444) di E. S. Piccolomini e le sue numerose traduzioni, la Carcel de Amor (1492) di Diego de San Pedro, il Processo de cartas de amores... di Juan de Segura (1548) e ancora, in ambito italiano, le raccolte di lettere del Bembo (1552), le Lettere amorose di Madonna Celia, gentildonna romana, scritte al suo amante (1562), le Lettere amorose di Girolamo Parabosco (1555) e, soprattutto, di Alvise Pasqualigo (1563). Cfr. C. KANY, The Beginnings of the Epistolary Novel in France, Italy and Spain, Berkeley, University of California Press, 1937, e L. VERSINI, Le roman épistolaire, Paris, P.U.F., 1979.
Sulla produzione epistolare cinquecentesca italiana e sulle traduzioni francesi si rinvia in particolare a A. QuONDAM (a cura di), Le "carte messaggiere". Retorica e modelli di comunicazione epistolare: per un indice di libri di lettere del Cinquecento, Roma, Bulzoni, 1981; J. BAsso, Le genre épistolaire en langue italienne (1538-1622): repertoire chronologique et analytique, Roma-Nancy, Bulzoni-Presses Universitaire de Nancy, 1990 e, dello stesso autore, Les traductions en français de la littérature épistolaire italienne aux XVIe et XVIIe siècles, in «Revue d'Histoire littéraire de la France», 78, 1978, pp. 906-918.

(9) Op. cit., pp. 227-230. 
fluttuante tra impostazione precettistica e autonomia creativa. All'interno di questi formulari può infatti verificarsi la presenza di uno o più nuclei narrativi composti da lettere legate da un rapporto consequenziale che ne costituisce una sorta di charpente narrativa. L'impianto precettistico risulta in questi casi alterato mediante la creazione di punti di fuga a coloritura romanzesca e cessa così di essere il solo elemento unificante dell'opera. Si può allora verificare il caso di testi manualistici con appendice antologica o di florilegi epistolari che svelano qualche più o meno evidente interferenza con la sfera della finzione narrativa.

Il presente studio intende addentrarsi in questo specifico campo di indagine, al fine di verificare le trasformazioni della scrittura epistolare e i suoi sconfinamenti nel territorio del romanzo. A tale scopo verrà posto in primo piano un meccanismo testuale che si può considerare indicatore della messa in atto di un processo, seppur ridotto, di mise en fiction: la coppia lettera-risposta. Trattandosi di una componente basilare della dinamica di interazione epistolare, di cui costituisce in un certo senso l'unità minima, essa può essere considerata come una sorta di nucleo generatore di ogni raggruppamento di lettere dotato di un qualche sviluppo narrativo.

Dal punto di vista semantico, la risposta va considerata come il complemento auspicato, se non necessario, del processo comunicativo che si instaura attraverso lo scambio epistolare. La lettera, e quella d'amore in particolare, in quanto discorso dell'assenza e sull'assenza ${ }^{10}$, instaura una dialettica paradossale tra gli interlocutori, fatta di simultaneità di presenza e assenza: l'amato, assente come referente, è presente come allocutore ${ }^{11}$ e la sua lettera di risposta diventa il luogo in cui l'assenza si fa presenza, in cui il destinatario non è più solo evocato, ma si manifesta come soggetto di una parola condivisa. La lettera d'amore, concepita come voce del desiderio, esige una risposta, in mancanza della quale si giunge ad una forma di estraniamento dall'altro e all'inaridimento della parola. Così come nell'esperienza vissuta, anche nel campo letterario, eccezion fatta per le forme epistolari monodiche strutturate come discorso di un'assenza che nel suo perenne rinnovamento si fa abbandono, la lettera di risposta è attesa e si configura come il completamento di un ciclo minimo di comunicazione. Si va allora dalla scrittura monofonica che ingloba in sé la traccia della risposta dell'interlocutore ${ }^{12}$ al dialogo epistolare vero e proprio. Ai margini del sistema letterario, nei manuali e nelle raccolte, predomina la giustapposizione di lettere modello, a cui tuttavia si affiancano, talvolta, brevi sequenze composte da una lettera e dalla relativa risposta. Da una forma di discorso stereotipato non individualizzato e avulso da ogni volontà di proiettare sul piano della finzione un'interazione tra gli interlocutori si passa in questi casi alla creazione di una situazione epistolare fittizia che riproduce lo scambio reale e induce un seppur minimo sviluppo narrativo della relazione amorosa.

Dal punto di vista delle dinamiche della comunicazione, l'articolazione dialogica lettera-risposta si afferma come asse strutturante della relazione intersoggettiva che si instaura tra due interlocutori epistolari e permette la piena realizzazione del carattere "transitivo" della lettera. L'inversione della formula elementare della comunicazione epistolare $^{13}$ (A scrive a B) trasforma il destinatario in destinatore (B scrive ad A) e

(10) La definizione della lettera come absentis ad absentem colloquium elaborata nel Medioevo (cfr. Histoire de la rhétorique dans l'Europe moderne (1450-1950). Publiée sous la direction de Marc Fumaroli, Paris, P.U.F., 1999, p. 349) si ritrova anche in Pierre Fabri, con una formula ripresa in seguito da Durand: «Et est a entendre que toute epistre ou lettre missive n'est aultre chose que aux absens parler comme presens [...]» (op. cit., p. 197).

(11) Cfr. R. BARTHes, Fragments d'un discours amoureux, Paris, Seuil, 1977, voce Absence; E.
LANDOWSKI, La lettre comme acte de présence, in La lettre. Approches sémiotiques, Actes du VIe Colloque Interdisciplinaire (Fribourg, 1984), Editions Universitaires de Fribourg, 1988, pp. 19-25 e P. VIOLI, Présence et absence. Stratégies d'énonciation dans la lettre, Ibid, pp. 27-35.

(12) E il «duo dont on entend une voix» di cui parla J. Rousset (Forme et signification, Paris, Corti, 1962 , p. 78), ridefinito «monodie dialogique» da J. HERMAN, op. cit., p. 86.

(13) Cfr. J. Herman, op. cit., pp. 84-85. 
avvia tra i due un processo di interazione che implica il costituirsi progressivo di una storia, semplice abbozzo nel caso di uno sviluppo minimo della coppia lettera-risposta, traccia narrativa più consistente nel caso di un'aggregazione composta da più missive. Soprattutto in presenza di carteggi di una certa corposità, i cambiamenti nel modo di porsi di un interlocutore rispetto all'altro diventano indice di un'evoluzione psicologica e, conseguentemente, di un avanzamento diegetico. Nelle compilazioni manualistiche avviene allora talvolta che gli interlocutori, da soggetti convenzionali di una forma di scrittura né autentica né fittizia, bensì semplice modello di realtà, tendano ad assumere contorni più individualizzati e a riprodurre dinamiche interattive reali, proiettandole al confine con l'immaginario.

Scendendo nel terreno concreto dell'analisi di precisi campioni testuali compresi in un arco cronologico che va dal secondo decennio del Cinquecento al primo quarto del secolo successivo, seguiremo l'evoluzione della lettera di risposta nelle sue diverse articolazioni, dalla più elementare (nucleo isolato di lettera e risposta) alle più complesse (nuclei numericamente più consistenti o addirittura dotati di una loro autonomia).

I secrétaires dell'epoca prevedono un certo spazio dedicato all'esemplificazione della risposta, sia nell'ambito delle lettere amorose che in altre tipologie epistolari. Prova ne sia il fatto che la presenza di lettres de réponse all'interno delle principali pubblicazioni manualistiche è talvolta segnalata fin dal titolo del volume. Dal già citato Maniere d'escrire par responce di Jean Quinerit de Mousne, rielaborato nel successivo Stile et maniere de composer, dicter et escrire toutes sortes d'Epistres ou lettres missives, tant par responce qu'autrement di Pierre Durand ${ }^{14}$, ripreso in parte a sua volta da Pierre Habert nel Miroir de Vertu ${ }^{15}$ e quasi integralmente nell'anonimo Nouveau

(14) L'opera conobbe otto edizioni concentrate in poco meno di trent'anni, il che dimostra il suo successo editoriale. Considerata, nell'ambito della produzione manualistica, la prima pubblicazione francese dotata di uno sviluppo ampio e sistematico, si rivela essere una sorta di composizione a mosaico che riunisce intere sezioni di opere precedenti. Vi si ritrovano infatti tracce del Prothocolle des secretaires e del trattato di Pierre Fabri. L'inserimento di una sezione intitolata La maniere d'escrire par response (pp. $53 \mathrm{r}^{\circ}-70 \mathrm{v}^{\circ}$ ), denota inoltre una chiara ripresa dell'omonimo volume di Quinerit de Mousne, a cui fanno seguito, inframmezzate dal testamento di Ciro e dall'arringa di Claudio Tolomei ambasciatore di Siena, Autres missives de plusieurs bons auteurs nouvellement adioustées (pp. $\left.84 \mathrm{r}^{\circ}-100 \mathrm{r}^{\circ}\right)$. Queste ultime si rivelano una fedele trascrizione delle lettere di Gabriello Simeoni originariamente contenute nell'Epitome de l'origine et succession de la Duché de Ferrare (Paris, $\mathrm{G}$. Corrozet, 1553). Il volume si conclude con De la ponctuation de la langue françoise seguito da Les accens de la langue françoise.

Per meglio far luce sulle complesse vicende editoriali dei primi manuali e sui molteplici collegamenti tra loro si consulti soprattutto $G$. GuEUDET, Archéologie d'un genre: les premiers manuels français d'art épistolaire, in Mélanges sur la littérature de la Renaissance à la mémoire de V.-L. Saulnier, Genève, Droz, 1984, pp. 87-98. Si vedano inoltre, A. Viala, La genèse des formes épistolaires en français et leurs sources latines et européennes. Essai de chronologie distinctive (XVIe-XVIIe siècles), in «Revue de Littérature Comparée», 2, 1981 , pp. 169-183 e M. DAumas, Manuels épistolaires et identité sociale, XVIe-XVIIe siècles, in «Revue d'Histoire moderne et contemporaine», 40, 1993, pp. 530-556. In particolare, il rapporto di filiazione del manuale di Pierre Durand con la Maniere d'escrire par responce di Quinerit de Mousne e con il Grant et Vray Art de pleine rhetorique di Pierre Fabri è stato studiato da C.L. LA CHARITÉ, art.cit. Si rinvia infine al repertorio bibliografico Y. GIRAUD e A.-M. Clin-Lalande, Nouvelle bibliographie du roman épistolaire en France. Des origines à 1842, Fribourg, Editions universitaires, 1995.

(15) Le Miroir de Vertu et Chemin de bien-vivre [...] Avec le Stile de composer toutes sortes de lettres missives, Paris, J. Caveillier, 1559 (edizione consultata: Paris, Micard, 1587). Il volume si compone di tre parti. La prima, Le Miroir de Vertu, contiene aneddoti riguardanti personaggi illustri dell'antichità, frammisti a sentenze e riflessioni morali. La seconda, formata dai tre libri dello Chemin de bienvivre, comprende quartine e distici morali. La terza è costituita dallo Stile de composer toutes sortes de lettres missives (pp. 103-140), raccolta di lettere convenzionali, mai firmate e sempre precedute da una rubrica contenente l'indicazione del destinatario e lo scopo della missiva.

Il più evidente modello di questa compilazione è stato identificato in Jean Le Moyne che pubblica a Parigi, nel 1556, L'instruction de bien et parfaictement escrire, tailler la plume et autres secrets pour se gouverner en l'Art d'Escriture, seguito da Plusieurs lettres missives (...). Sugli stretti rapporti tra le due 
stile del $1584^{16}$, si giunge, se non ad una vera e propria codificazione, quantomeno alla creazione di un vasto repertorio di lettere di risposta, presentate come volet conclusivo di un dittico comunicativo. Quasi trent' anni prima, del resto, lo stesso Pierre Fabri aveva sentito la necessità di inquadrare da un punto di vista teorico la lettera di risposta, distinguendo «deux manieres de lettres missives: les unes missives actives, les autres missives responsives. Les missives actives s'appellent celles qui sont narratives, et petitores et comminatives. Les missives responsives s'appellent les celles qui doibvent response en acordant, excusant, congratulant, etc.» ${ }^{17}$. Questo abbozzo di teorizzazione non avrà tuttavia particolare seguito fino al secolo successivo, quando Puget de La Serre, nel suo fortunatissimo Secretaire à la mode ${ }^{18}$, presenta una classificazione di diversi tipi di lettere tra cui inserisce anche le Lettres de response. Le riconosce tuttavia solo in parte come tipologia autonoma in quanto conseguenti alla lettera che le precede:

Finalement est à observer, qu'encor que les enseignemens precedens regardent principalement les lettres qu'on escrit le premier, sans avoir esté provoqué par celles d'un autre; toutesfois il est facile de les accomoder aux lettres de responce de sorte qu'il n'est point besoin d'en traitter à part. Celles à qui on respond en prescrivent la matiere, \& n'est requis autre chose que d'y satisfaire de point en point, selon que nous en avons le moyen ou que la prudence nous le permet. Es lettres d'affaires il ne faut laisser passer sans reponse aucune chose qui merite. En celles de compliment il n'est pas necessaire d'estre tousjours si exact. [...] mais tant és unes qu'és autres la promptitude est fort à priser (pp. 30-31).

Ad esclusione di questi due tentativi di inquadramento teorico della risposta, prevale, nella maggior parte delle raccolte e dei manuali presi in esame, l'esemplificazione diretta di questo tipo di lettera. Avviene così talora che, in un insieme epistolare spesso eterogeneo formato da elementi giustapposti secondo pure finalità didatticoesplicative e perciò slegati gli uni dagli altri, risaltino alcune coppie formate da lettera e relativa risposta al cui interno si condensano e trovano uno sviluppo elementi narrativi elementari.

Nella Nouvelle maniere d'escrire par responce di Jean Quinerit de Mousne al cui interno sono annunciati, fin dal sottitolo, «la Rhetorique Ciceroniane, avec plusieurs

opere cfr. E. COURBET Deux poètes professeurs d'écriture au XVIe siècle: J. Lemoyne et P. Habert, in «Bulletin du bibliophile et du bibliothécaire», 1911, pp. 101-107; 143-153; 316-327.

(16) Nouveau stile et maniere de composer, dicter, er escrire toutes sortes d'epistres, ou lettres missives tant par responce qu'aultrement. Plus les lettres amoureuses des Amans passionnez Paris, Nicolas Bonfons, 1584. Si tratta di un rifacimento piuttosto preciso dello Stile et maniere... di Durand che riprende inoltre alcune lettere contenute anche nel Miroir de vertu.

Il volume è composto da una serie di indicazioni riguardanti la redazione delle lettere e la disposizione della materia epistolare all'interno delle diverse tipologie presentate. La trattazione teorica di ogni singolo argomento è illustrata da numerosi esempi. Scriventi e destinatari sono spesso indicati mediante la loro categoria di appartenenza (re, signore, dama, padre, madre, avvocato, medico, capitano...). Nei casi in cui la loro identità è precisata, si tratta di un'identità reale, spesso di personaggio storico (Pitagora, Cesare, Scipione, Poliziano, Pico della Mirandola, Lorenzo de' Medici...). Vi è anche, ripresa dal Fabri attraverso la mediazione del Du- rand, ma non fedelmente tradotta dal Piccolomini, una lettera di Eurialo a Lucrezia, rubricata nella categoria Lettres vicieuses.

Le Lettres amoureuses des amans passionnez recueillies de divers Autbeurs, inserite in appendice (pp. $93 \mathrm{r}^{\circ}-130 \mathrm{v}^{\circ}$ ), sono lettere scritte da mano maschile («l'Amant», «l'amy», «un Gentihomme»). Ripropongono situazioni amorose stereotipate, sono slegate tra loro e mai accompagnate da risposta. Le prime due lettere ripropongono ampi frammenti di una lettera tratta dal manuale di Quinerit de Mousne e a sua volta inglobata dal Durand. Altri probabili emprunts tratti da fonti diverse restano da verificare.

(17) Op. cit., pp. 197-198.

(18) Le Secretaire à la mode ou Methode facile d'escrire selon le temps diverses lettres de compliments, amoureuses et morales. La prima edizione è datata 1640 (Paris, O. de Varennes); l'edizione consultata, pubblicata a Amsterdam nel 1665 per i tipi di J. Jansson e E. Weyerstraet, è stata localizzata alla Biblioteca Querini Stampalia di Venezia. Si presenta arricchita da «un Recueil des Lettres morales des plus beux esprits de ce temps. Et des Complimens de la Langue Françoise». 
belles acclamations lesquelles font les amans l'un à l'aultre», non tutte le lettere sono accompagnate da risposta, né sono ordinate secondo un preciso criterio. Il materiale presentato illustra numerose categorie epistolari tra le quali la lettera d'amore è rappresentata, a dispetto del sottotitolo, solo da tre esempi su cinquantuno. Le missive, precedute da una brevissima rubrica che ne sintetizza la finalità e fornisce le coordinate essenziali dell'atto comunicativo ${ }^{19}$, non hanno collegamenti tra loro se non nel caso delle coppie con risposta, che risultano essere soltanto quattordici. Tra queste, la più interessante ai fini della nostra indagine sembra essere l'ultima, intitolata L'amant a sa dame par amour avecques la responce de la dame. Insieme alla missiva seguente, scritta nuovamente dall'amante, essa forma un breve raggruppamento di tre lettere concatenate. Unico caso in tutto il volume, questa amplificazione della coppia lettera-risposta indica, rispetto agli altri binomi epistolari contenuti nell'opera, l'infittirsi dei legami consequenziali e l'evidenziarsi di alcune implicazioni narrative. Due delle lettere in questione risultano inoltre firmate e dunque ricondotte ad una, seppur vaga, individualità dello scrivente. Il nome che compare è quello dell'innamorato («le vostre humble serviteur Floridus»), da un lato assimilato, attraverso la formula «vostre humble serviteur», alla schiera indistinta di firmatari di lettere amorose modello, dall'altro individualizzato mediante l'attribuzione di un nome, per quanto convenzionale. L'originalità di questo trittico epistolare è tuttavia solo apparente. In realtà è proprio l'attribuzione del nome allo scrivente a costituire l'aggancio che ci permette di scavare nel tessuto stratificato di questo formulario e di identificare le tre lettere con altrettante missive inserite in un'opera di finzione antecedente: il Jardin amoureux di Christophe de Barrouso ${ }^{20}$. In questa breve composizione romanzesca in versi di impronta allegorica, che non godette peraltro all'epoca di particolare successo, la narrazione è intercalata da 14 lettere in prosa scritte sia dall'innamorato che dall'amata. La parte utilizzata nella Maniere d'escrire par response è unicamente quella epistolare e riguarda per intero le prime due lettere e parzialmente la terza. Il Jardin amoureux si rivela una fonte non trascurabile non tanto per l'apporto quantitativo, in realtà non molto esteso, quanto piuttosto per la scelta operata da Quinerit de Mousne di concludere il suo manuale conferendogli un certo spessore diegetico che egli affida alle potenzialità aggreganti della risposta. Mutuando una sequenza epistolare da un testo appartenente al campo della finzione, l'autore trapianta in un'opera compilativa di natura precettistica un segmento narrativo, facendolo figurare come una possibile applicazione del meccanismo dialogico epistolare. Mediante l'inserzione di queste tre lettere, l'insieme slegato, se non eterogeneo, di modelli epistolari si addensa alla fine in un nodo narrativo in cui alcuni elementi fittizi, pur non originali, si articolano in un intreccio sostenuto unicamente dalla funzione affabulatoria della lettera di risposta.

Intessuta di clichés espressivi attinti dal repertorio linguistico e metaforico della prosa amorosa, la prima lettera è sostanzialmente un'appassionata dichiarazione d'amore in cui l'innamorato, soggiogato dalla bellezza dell'amata e sottomesso alla

(19) Ricorre spesso la formula: «Comment tu peux...» seguita dall'indicazione dell'atto comunicativo oggetto della missiva (ringraziare, raccomandare qualcuno, chiedere un favore, annunciare la morte di un amico, consolare...). Sempre è precisata la categoria (sociale o familiare) di appartenenza del destinatario (banchiere, avvocato, mercante, principe, dama, amico, figlio, padre...); il mittente può essere indicato secondo le stesse modalità del destinatario (gentiluomo, banchiere, madre, figlia, amante) oppure indicato genericamente con un «tu», oppure infine assimilato ad un'identità puramente virtuale mediante il ricorso a formule come
«Parlement devant...», cui fanno seguito «ung Roy», «ung Pape», «un juge criminel», ecc.

(20) CH. DE Barrouso, Le jardin amoureux contenant toutes les regles d'amours: avecques plusieurs lettres missives tant de l'amant comme de l'amye, [s. n.], [s. d.]. Fu probabilmente pubblicato da Claude Veycellier tra il 1528 e il 1530. Cfr. Y. Giraud, L'étrange «Jardin amoureux» de Maître Barrouso, in Conteurs et romanciers de la Renaissance. Mélanges offerts à G.-A. Pérouse. Etudes recueillies et présentées par J. Dauphiné et B. Périgot, Paris, Champion, 1997, pp. 209-224. 
sua volontà, le offre i suoi servigi, implora benevolenza e chiede «une benigne responce», unico possibile rimedio alle sue sofferenze. La risposta sdegnata della dama non concede speranze allo spasimante, a cui viene proibito di scrivere ancora tali «abhominables lettres». La replica finale ci mostra Floridus che, in preda alla disperazione, si augura la morte, ma, riconoscendosi incapace di non amare la sua bella crudele e di rinunciare a scriverle, conclude affermando che il suo tormento potrà essere lenito solo dalla concessione di essere il suo «loyal amant et serviteur».

Risalta da questo breve scambio epistolare una particolare attenzione alla materialità della scrittura e alle modalità concrete dell'invio e della ricezione, elementi essenziali per ogni corrispondenza reale, e ancor più per quelle fittizie. Nei romanzi epistolari sei-settecenteschi essi sono infatti spesso posti in primo piano e fatti oggetto di una rappresentazione verbale che ha lo scopo di alimentare l'illusione di veridicità. Nel nostro testo, oltre ai ripetuti accenni alla consegna delle lettere da parte di un messaggero e alle conseguenti reazioni del destinatario, vengono più volte rappresentati da entrambi gli interlocutori gli atti di scrittura e di lettura e le loro implicazioni soggettive. Si rende così più verosimile il carteggio e si crea inoltre un alone psicologico che, in quanto riflesso dell'atto comunicativo, ne mette maggiormente in luce le dinamiche interpersonali, determinando da un lato un ispessimento del legame sentimentale fra i protagonisti e, dall'altro, una crescente attesa da parte del lettore che si interroga sui possibili sviluppi della storia nascente.

Scrive Floridus:

Et d'aultre part voyant la nullité et insuffisance de mes precedens services envers vous la tremblante main la plume pour le blanc papier vergier ne peult sotenir [...] (f० 54$)$.

\section{Gli risponde l'amata:}

[...] en regardant le contenu de [vos abhominables lettres] a grant peine mon ire ay sceu refrener et moy abstenir de les dessirer en pieces. [...] Et a celle fin que vous ne soyez si hardy de plus en ce cas continuer et sachez que ie ne suis icelle a qui telles abusives lettres se doivent transferir ne envoier, i'ay voulu contre ma coustume vous rescrire ceste presente laquelle mon courage a grant peine pouvoit souffrir de achever $[\ldots]$ ( $\left.f^{\circ} 56\right)$.

\section{A cui fa eco l'innamorato:}

Et d'aultre part, voyant chose en laquelle vous candides et plaisantes mains avoyent touché, tant de ioye en moy abondoit que les apetissans yeulx de les regarder et la revifiée langue de plusieus foys les eloquens epitetz d'icelles relire nullement se pouvoyent abstenir ne divertir; toutesfoys voyant icelles du exhuberant flume de larmes de mes pleurans yeulx emanant estre madides et irriguées de paur de en tel estat les consumer i'ay mon ardant appetit moderé et refrené veuillant icelles empres mon cueur tousiours porter en signe de veneration et honneur $\left(f^{\circ} 57\right)$.

Questi ed altri precisi riscontri tra lettera e lettera rendono l'insieme coeso e coerente dal punto di vista dell'articolazione discorsiva e rafforzano i meccanismi interattivi. Il dialogo scritto dà voce al discorso amoroso e instaura tra lettera e lettera un legame di continuità e necessità. La risposta implorata dall'amante e concessa, seppur a malincuore, dall'amata assume infatti il valore di una compensazione del desiderio e, come tale, diventa elemento assolutamente indispensabile al permanere della passione. La comunicazione epistolare si definisce così come una sorta di trasposizione linguistica della relazione d'amore e la risposta, che permette il rilancio del dialogo, diventa, oltre che necessario completamento dell'atto comunicativo, importante elemento di concatenazione narrativa. 
Si leggano a conferma di ciò i punti di raccordo fra le tre lettere:

Et puis vous estes la cause de tel mal et grief martire et vous seulle le povez guerir et alleger; vueillez porriger le vray remede en envoyant une benigne responce laquelle tres affectueusement desire et attens (L'amant a la dame par amour, $\mathrm{f}^{\circ} 55$ ).

Tant desmesurement a vostre insolent messagier mon pensement troublé que ie ne puis assez esbahir en imaginant quelle cause vous [...] donne si presumpteuse hardiesse de mon acostumé repos par vos abhominables lettres et parolles vexer et trobler [...]; se plus en ceste matiere perseverez a moy ferez chose desplaisante [...] Pourquoy ie vous prie [...] vueillez a ma requeste condescendre en laquelle chose a moy ferez singulier plaisir. En vous disant a Dieu (Responce de la dame, f $\mathrm{f}^{\circ}$ 5-56).

Et quant à ce que dictes avoir eu vouloir de mes lettres dessirer et le messagier mal traicter suffire devroit ce que vostre seul regard et souvenance en moy continuellement lacerant et estrassinant iournellement exercent sans ce que vos tendres mains de telle cruaulté voulsissiez maculer. Et quant a ce lequel m'est plus grief et amer que moy mandez de non plus vous escripre ne continuer en mon propos, sachez ma dame qu'ainsi que nulle personne pourroit vostre grant beaulté de vous separer, ainsi aultre que la mort ne scauroit mon propos et vouloir entrerompre ne diminuer [...] (L'amant a la dame par amour, f $\mathrm{f}^{\circ}$ 57).

Pur senza giungere all'elaborazione di un testo fittizio, Quinerit de Mousne si appropria di un frammento romanzesco scritto da altri sfruttandolo come esemplificazione di una possibile modalità di risposta epistolare. Fermo restando l'intento dimostrativo della composizione, il vago tracciato di una storia di cui si tacciono l'origine e gli sviluppi prende forma nelle ultime pagine del libro rivelando l'insinuarsi di un'ambigua sfumatura di finzione.

Anche nell'ambito della lettera familiare si riscontra qualche esempio di segmento narrativo costituito dallo scambio minimo lettera-risposta. Questo avviene in particolare nel Miroir de vertu, nello Stile et maniere di Durand e nel Nouveau Stile, che presentano frammenti di dialogo epistolare molto ridotti, sviluppati quasi esclusivamente in sequenze di due soli elementi ${ }^{21}$. Entrambe le opere evidenziano fin dalle pagine prefatorie un marcato intento educativo. Si presentano infatti ai lettori come strumenti didattici volti a «rendre un chacun diligent lecteur, en adioustant le don de sa naturelle grace, honneste et expert Secretaire» ${ }^{22}$ e ad apprendere a «bien forger comme a l'enclume missives ${ }^{23}$. Ponendosi come complemento di compilazioni epistolari più dotte e meno accessibili ad un pubblico non erudito, privilegiano la formazione del «vulgaire ignorant, lequel veut dresser missives» ${ }^{24}$. Nonostante queste premesse di natura esclusivamente pedagogica, qualche coppia di lettere riesce a tratteggiare una cornice evenemenziale di riferimento e a fornire alcuni indizi di concatenazione tra i fatti evocati, così da conferire in alcuni punti al testo una dimensione diacronica funzionale all'instaurarsi di un embrione narrativo. In particolare due brevi scambi tra moglie e marito e tra madre e figlia contenuti nello Stile et maniere

(21) In Habert si trova un'unica serie di tre lettere, costituita da due lettere $A$ une jeune Damoyselle, laquelle tu esperois d'avoir en mariage (pp. $135 \mathrm{r}^{\circ}$ $135 \mathrm{v}^{\circ}$ ), l'ultima delle quali è seguita dalla Responce de ladite Damoyselle (pp. $136 \mathrm{r}^{\circ}-136 \mathrm{v}^{\circ}$ ). Si tratta però di un'aggregazione epistolare caratterizzata da un'esclusiva finalità esemplificativa, priva di ogni connotazione psicologica degli scriventi. La stessa formula usata nel titoletto risente in maniera evidente della preoccupazione essenzialmente didattica del compilatore, che ricorre ad una generica e spersonalizzante seconda persona singolare.

La coppia di lettere che segue questo breve scambio, ripresa peraltro una trentina d'anni dopo nel Nouveau stile..., ripropone un'identica situazione comunicativa e dei contenuti analoghi, il che non $\mathrm{fa}$ che accrescere la dimensione stereotipata del rapporto epistolare in questione.

(22) Nouveau stile et maniere..., Aux lecteurs.

(23) Le Miroir de Vertu ..., Au lecteur. Sonnet.

(24) Nouveau stile et maniere..., Aux lecteurs. Salut 
e ripresi in toto nel Nouveau stile offrono qualche elemento di un certo interesse e si allineano in una direzione che vagamente accenna a un quadro narrativo e a suoi possibili sviluppi.

Nel primo la moglie scrive al marito, assente da lungo tempo, e lo rimprovera per averle scritto solo due brevi lettere in un anno e per averla lasciata sola con due figli in tenera età. Bisognosa delle sue attenzioni, aggiunge poi che:

Outre ce, ne vous oubliez que ie n'ay mes aises de chose aucune de la vie necessaire, mais sachez que ie suis femme, \& ieune, ne pire ne meilleure que les autres; donc vous supply $\&$ requiers vous vouloir d'icy approcher, car nous avons grand besoing de vous [...] (Stile et maniere, p. $58 \mathrm{v}^{\circ}$; Nouveau stile, p. $66 \mathrm{v}^{\circ}$ ).

La risposta del marito riprende punto per punto la lettera ricevuta. Gli stessi accenni fatti dalla moglie alla scarna corrispondenza con il consorte si ritrovano nella risposta di quest'ultimo, che fa riferimento a più lettere «fort lamentables» indirizzategli dalla donna e che giustifica la scarsa frequenza dei suoi scritti con le difficoltà nella consegna della posta in posti lontani:

[...] vous croyez paraventure qu'icy où ie suis en Lyon, soit iusques d'Amboise autant de chemin, comme de Bloys à Tours. En verité ie vous ay escrit pour le moins neuf fois l'an: \& plus souventesfois avient, comme vous pouvez considerer, que par negligence des porteurs les lettres perissent [...] Dieu aidant dans trois mois i'espere estre pres de vous. (Stile et maniere, p. $59 \mathrm{r}^{\circ}$; Nouveau stile, p. 67)

Nel complesso il breve scambio fra coniugi fornisce alcune coordinate narrative elementari: indicazioni spaziali e temporali, evocazione di alcuni tratti personali degli interlocutori e del loro contorno familiare, riferimenti all'intrecciarsi della relazione epistolare, anche negli aspetti più concreti della trasmissione delle lettere. Tuttavia, in mancanza di uno sviluppo adeguato, queste tracce di un vissuto costruito nello spazio ridotto di due lettere testimoniano un'articolazione narrativa destinata a rimanere allo stato embrionale.

Sullo stesso piano, ma con una maggiore incidenza di indizi potenzialmente finzionali, si situano le due lettere scambiate tra una madre e la giovane figlia vedova di nome Antoinette. Anche in questo caso sono presenti accenni ad una corrispondenza già avviata da tempo e precisi toponimi. In più, sono inseriti accurati ritratti di entrambe le interlocutrici e dettagliati riferimenti, anche psicologici, ad altri personaggi, una vicina della figlia e suo fratello:

Parquoy escoutez, ie vous prie ma treschere mere, vous cognoissez bien Ieanne, laquelle a un frere aagé de vingt-quatre ans, yvrogne, mal apprins, ioueur \& gourmand, deux ans y a, $\&$ plus, que celuy par lettres, dons \& promesses deshonnestement me va tentant et ce ne faict tant pour son desir, que par la mauvaise volunté de sa seur Ieanne, laquelle estant desormais de trente-six ans, et richement mariée, mais à homme vieillard, à qui en demande est de son amour tresliberale (Stile et maniere, p. $62 \mathrm{v}^{\circ}$; Nouveau stile, pp. $71 \mathrm{v}^{\circ}-72 \mathrm{r}^{\circ}$ ).

Se ne ricava una breve storia, suscettibile di possibili sviluppi, in cui si intrecciano gelosie tra giovani vicine, disegni disonesti, preoccupazioni e consigli materni e dichiarazioni di onestà d'intenti da parte della figlia. Alcuni degli attori di questo quadro di vita provinciale sono inoltre dotati di un nome, oltre che di un'età ben definita e di uno stato civile, il che concorre a creare non trascurabili presupposti all'emergenza della finzione e alla costruzione di un canovaccio narrativo.

A conclusione di questo primo gruppo di esempi si può affermare che, se la coppia lettera-risposta si configura come matrice narrativa elementare, ciò avviene 
perché questo scambio ridotto all'essenziale è però in sé concluso e riconducibile ad una micro-situazione comunicativa dotata di una cornice referenziale al cui interno si instaura una dinamica evenemenziale. Lo sviluppo delle lettere di risposta all'interno di testi manualistici ove spesso si rinvengono echi o riprese letterali di altre opere analoghe incrementa la circolazione di materiali epistolari comuni e di formule stereotipate. Si consolida in questo modo una sorta di patrimonio collettivo costituito da un repertorio di clichés espressivi e di situazioni convenzionali predeterminate, utilizzato a scopo didattico e destinato a tracciare il quadro di riferimento di varie tipologie di lettere, in primis di quella d'amore. Questo spiega la scarsa originalità formale riscontrabile nella maggior parte di lettere e risposte presenti nei manuali, che molto di rado danno vita a scambi dalle potenzialità narrative significative. Queste pur rare occorrenze costituiscono tuttavia un indice non trascurabile delle capacità agglomeranti della lettera e contribuiscono a dare l'avvio ad un percorso di narrativizzazione di materiali epistolari originariamente concepiti come disomogenei. Tale percorso, appena percepibile nel corso del Cinquecento, sarà distinguibile più chiaramente nel secolo successivo, epoca in cui il discrimine tra il manuale, o più ancora la raccolta di lettere, e l'opera di finzione si fa meno netto e non di rado si assiste allo sviluppo del romanzo epistolare per filiazione diretta dai secrétaires.

Una situazione più favorevole all'aggregarsi di nuclei narrativi di maggior consistenza si ritrova nell'ambito di quelle raccolte non accompagnate da annotazioni teoriche, composte perciò da insiemi epistolari non caratterizzati da un esplicito intento normativo e catalogatorio. A differenza dei manuali, governati da principi classificatori che inducono ad un ordinamento delle lettere secondo la loro categoria di appartenenza e condizionati dalla necessità di articolare le singole missive in base al principio retorico di cui esse costituiscono l'applicazione, le raccolte sono rette da una finalità puramente illustrativa. Si tratta infatti di florilegi di lettere d'amore o familiari o morali composte da uno o da diversi autori. In quest'ultimo caso accade spesso che l'identità degli autori non sia resa nota mediante l'indicazione dei loro nomi, ma mediante un accenno, nel titolo, al loro riconosciuto valore letterario ${ }^{25}$.

Un caso particolare è costituito dalle raccolte di Herö̈des, lettere la cui maggior fortuna editoriale si estende in Francia dal primo Cinquecento alla metà del secolo seguente. Essa è dovuta innanzi tutto alle numerose traduzioni, integrali o parziali, delle Herö̈des di Ovidio da parte di autori come Octovien de Saint-Gelais ${ }^{26}$, Charles Fontaine $^{27}$ e, nel secolo successivo, Du Perron ${ }^{28}$, Claude Gaspar Bachet de Méziriac ${ }^{29}$ e Pierre de Deimier ${ }^{30}$, a cui si deve la prima traduzione integrale in prosa. Un altro importante fattore che contribuisce al notevole successo di queste lettere d'amore scritte da personaggi mitologici è costituito dall'instaurarsi di una tendenza parallela a quella della traduzione: l'imitazione delle epistole ovidiane ripresentate nella veste di risposte date dal destinatario. Fin dalla metà del Cinquecento si assiste all'elaborazione di queste false Heroides che tendono nel corso del tempo a staccarsi sempre

(25) Si ritrovano spesso formule fisse che tendono a moltiplicare l'istanza autoriale, presentando le lettere, a volte indebitamente, come una composizione a più mani. È il caso delle Lettres amoureses et morales des beaux esprits de ce temps di François de Rosset (Paris, L'Angelier, 1609), delle Lettres amoureuses de divers autheurs de ce temps di Georges de Scudéry (Paris, Courbé, 1641) o ancora de Les plus belles lettres françoises sur toutes sortes de sujets, tirées des meilleurs auteurs di Pierre Richelet (Paris, Brunet, 1698). 1500 .
(27) Les Epistres d'Ovide nouvellement mises en vers François par M. Charles Fontaine [...] Plus y a la response à icelles epistres, Lyon, Jean Temporal, 1552.

(28) Plainte de Pénélope à Ulysse, in Les Muses raillées, Paris, Guillemot, 1603.

(29) Les epistres d'Ovide traduites en vers français. Avec des commentaires fort curieux. Première partie, Bourg-en-Bresse, J. Taintureir, 1626.

(30) Lettres amoureuses, non moins pleines de belles conceptions que de beaux désir, ensemble la traduction de toutes les epistres d'Ovide, Paris, Sevestre, 1612. 
più dalla matrice originale per acquisire caratteristiche autonome avvicinandosi progressivamente, grazie soprattutto al passaggio dai versi di ascendenza ovidiana alla prosa, a modelli di lettere amorose ${ }^{31}$. Nell'impossibilità di esaminare in questa sede una produzione così vasta, ci soffermeremo sulla prima di queste continuazioni delle Heroides, quella redatta da Michel d'Amboise e inserita nella raccolta di Charles Fontaine pubblicata nel 1552.

La traduzione delle prime dieci Heroides è seguita dalla Response aux dix epistres precedentes par Michel d'Amboise. Queste ultime sono tratte dalle Contrepistres d'Ovide nouvellement inventees et composees par l'Esclave Fortuné (Paris, Janot, 1541), scritte dallo stesso Michel d'Amboise, mosso, almeno in parte, da un atteggiamento critico nei confronti di Ovidio a cui rimprovera una troppo sentita difesa dei personaggi femminili. L'intervento sul testo ovidiano denota nelle Contrepistres una marcata impronta antifemminile e si pone come difesa dell'orgoglio maschile. Nella ripresa che ne fa Charles Fontaine dieci anni dopo l'inserimento delle risposte viene diversamente motivato, privato di ogni intento polemico e finalizzato unicamente ad accrescere il piacere della lettura:

II nous a semblé qu'il ne viendroit point mal à propos d'adiouster aux Epistres precedentes la response qui s'ensuit, nonobstant qu'elle soit d'une autre main, tant pour faire le livret plus ample, que pour vostre recreation (Aux lecteurs, p. 237).

È interessante osservare la trasformazione subita dall'originale ovidiano, in cui ogni lettera si configura come un canto solitario privo di ogni volontà comunicativa e retto soltanto da finalità puramente espressive e retoriche. La costruzione delle risposte denota un'operazione di recupero e di valorizzazione degli elementi narrativi contenuti nelle beroides. In virtù delle loro qualità aggreganti, essi vengono ripresi e ampliati nella replica che diviene così una sorta di complemento narrativo dell'epistola originale. La risposta si sviluppa quasi per gemmazione e, sfruttando le potenzialità della lettera a cui fa seguito, ne espande i confini. Prende forma così un dittico epistolare inquadrato in un'ottica narrativa che ne fa emergere con maggiore evidenza le caratteristiche di invenzione letteraria.

Il raddoppiamento dell'epistola originale non si configura certamente come un tratto di originalità attribuibile al suo traduttore cinquecentesco. Ovidio stesso ricorre infatti, anche se tardivamente, ad un ampliamento del monologo mediante una sorta di eco che gli attribuisce una maggior risonanza, come testimonia l'aggiunta, alla fine della raccolta, di tre epistole di risposta ${ }^{32}$. L'inserimento di altre repliche, diverse da quelle ovidiane, da parte di Charles Fontaine tende però ad estendere sistematicamente lo schema dialogico all'intera raccolta, aprendo la strada alla fortunata produzione delle false beroides cinque-seicentesche. La tendenza a "romanzare" insiemi di lettere di riconosciuto valore letterario mediante l'aggiunta di risposte emerge del resto a più riprese nel corso dei secoli. Dalle Responses di Michel d'Amboise alle Réponses alle Lettres portugaises, opera distante, ma non del tutto estranea per tipologia enunciativa alla composizione ovidiana, assistiamo infatti al manifestarsi di un'esigenza di inventare continuazioni di testi epistolari monodici. In questi casi l'ideazione della risposta, se da un lato può essere sentita come un'appendice prosaica che mutila in un certo senso lo slancio poetico, dall'altro si pone come complemento

(31) Cfr. D. Dalla Valle Dalle "Heroides" di Ovidio alle lettere eroiche in Francia nel XVI $e$ XVII secolo, in «Studi Francesi», 119, 1996, pp. 307-316.

(32) Le ultime tre coppie di epistole (Paride a Elena e Elena a Paride; Leandro ad Ero e Ero a
Leandro; Aconzio a Cidippe e Cidippe ad Aconzio) hanno posto per lungo tempo un problema di attribuzione, anche a causa del loro più ampio sviluppo e di diverse caratteristiche stilistiche. In realtà sono da considerarsi autentiche, anche se composte più tardi (Cfr. A. Della Casa, Introduzione a Publio 
che trasforma il monologo in dialogo epistolare, conferendo una maggior verosimiglianza alla comunicazione, ma proiettandola nel contempo quasi paradossalmente nella sfera della finzione letteraria. Nella sua duplice veste di conclusione in tono minore e di elemento déclencheur di un intreccio latente, la risposta sembra farsi interprete di una segreta deriva finzionale che muove, almeno in parte, la scrittura epistolare fin dagli albori.

A conclusione della nostra indagine restano da considerare ancora due casi: l'alternanza lettera-risposta all'interno di una scambio epistolare inserito in una raccolta e l'uso della risposta come testimonianza parziale in una relazione epistolare di cui viene reso leggibile solo uno dei poli della comunicazione.

Il primo caso sarà illustrato da una delle raccolte di maggior successo di tutto il Seicento: Le Secretaire de la Cour di Puget de la Serre ${ }^{33}$. La sezione dedicata alle Lettres amoureuses, molto composita ${ }^{34}$, contiene numerosi modelli di lettere di risposta ${ }^{35}$ ed è per di più disseminata di nodi narrativi, spesso imperniati sulle risposte. Il percorso dell'invenzione è in questi luoghi del testo particolarmente evidente. Si possono infatti rilevare due nuclei aggreganti, uno molto ristretto, l'altro piuttosto ampio. Il primo è costituito da una serie di tre lettere, la prima delle quali viene presentata come l'ennesimo esempio di Lettre pour demander response. Al titolo questa volta però viene aggiunta una precisazione («après avoir escrit plusieurs fois») che evoca un passato di scrittura a cui la lettera si collega per poi richiamare a sé la tanto attesa risposta, a cui farà infine seguito un'ulteriore replica. L'esigenza esemplificativa propria del formulario abbandona qui lo schema della sequenza enumerativa e si esplica invece attraverso la disposizione delle lettere-modello su un asse temporale, elemento costitutivo di ogni storia. L'attribuzione di nomi, Diana e Silvandre, ai due interlocutori si somma ai cenni referenziali che appaiono sullo sfondo e alimenta così l'impressione del lettore di sconfinare nel terreno della finzione. Questa stessa impressione si avverte in maniera più decisa nella lunga serie di lettere a Clorinde che Puget de La Serre dissemina nella sua raccolta, intercalandole con altre di diverso genere, ma circoscrivendone la maggior parte sotto il titolo di Lettres particulieres de l'autheur à Clorinde. I topoi della lontananza, della bellezza e crudeltà dell'amata e infine della sottomissione dell'amante scandiscono il succedersi di questi frammenti di corrispondenza che ripercorre il tracciato canonico della relazione amorosa tendre, pur senza fornire un vero e proprio raccont ${ }^{36}$. Le lettere di Silvandre (così si firma l'autore con un evidente richiamo letterario) a Clorinde sono organizzate in uno scambio dapprima serrato e cadenzato da un'alternanza regolare di lettera e relativa risposta. Si diradano in seguito, disperdendosi all'interno della raccolta a volte in coppie sequenziali, a volte isolatamente, intercalate da

Ovidio Nasone, Opere, vol I, Torino, Utet, 1982, pp. 209-219.

(33) Le Secretaire de la Cour, ou la maniere d'escrire selon le temps. Dedié a Monsieur de Malherbe. Par le Sieur de la Serre, Paris, Pierre Billaine, 1624. Si segnala che una copia dell'edizione citata (in - $8^{\circ}, 487$ pp.) è stata localizzata alla Biblioteca Querini Stampalia di Venezia (I. G. 1060) e va così ad aggiungersi a quella conservata nella Bibliothèque cantonale et universitaire di Friburgo (Svizzera) segnalata Y. GIRAud e A.M. Clin-LalanDE in Nouvelle bibliographie du roman épistolaire en France, cit., p. 34.

Questa prima edizione costituirà il nostro testo di riferimento.

(34) Vi si susseguono brevi serie di Lettres de presentation de service, di Lettres plaintives, di Lettres d'un Amant à sa Maistresse sur son absence, e, isolate, lettere «de remonstrance a sa Maistresse», «sur l'inconstance», «sur la rigueur», ecc.

(35) Responses pour les Dames aux lettres d'offre de service, Lettres pour demander response, Lettres des Dames pour respondre.

(36) J. Chupeau parla di «fiction épistolaire dans le goût du roman sentimental», riferendosi in particolare ad un'edizione successiva (Paris, P. Billaine, 1631), che non ci è stato possibile consultare, al cui interno è segnalata una continuazione delle «Douze Lettres particulières de l'Autheur à Clorinde, avec leur response» (Puget de La Serre et l'esthétique épistolaire: les avatars $d u$ «Secrétaire de la Cour», cit., p. 118). Gli fa eco Y. Giraud che riconosce alle lettere a Clorinde le caratteristiche di «un petit roman sentimental, débarassé de toutes péripéties extemes et de circonstances concrètes» (De la lettre à l'entretien: Puget de La Serre et l'art de la conversation, in Art de la lettre, art de la conversation à l'époque classique en France, cit., p. 221. 
lettere appartenenti ad altre tipologie. Non siamo in presenza di un'autentica articolazione romanzesca quanto piuttosto di una successione di segmenti narrativi sorretti al loro interno da una coerenza e da una consequenzialità indubbie, ma non sufficientemente omogeneizzati gli uni con altri perché carenti dal punto di vista degli elementi referenziali e dell'intreccio. Ancora una volta è dunque essenzialmente la risposta a figurare come legame che garantisce una forma di coesione interna in un dialogo epistolare che si ferma alle soglie della finzione letteraria.

Mettiamo qui di seguito in evidenza alcuni snodi particolarmente significativi di questa conversazione galante in forma epistolare, allo scopo di sottolineare come il movimento narrativo tenda ad essere ritmato da un'alternanza di lettere maschili centrate sul topos dell'amante sottomesso e della donna amata bella e crudele e di repliche femminili animate da uno spirito quasi corrosivo che, mettendo in discussione lo stereotipo dell'amant martyr, crea uno scarto comunicativo in grado di rilanciare il dialogo. Le risposte di Clorinde sdrammatizzano i toni di Silvandre e consegnano al lettore un'immagine più mossa e meno monocorde del rapporto d'amore tra gli interlocutori. Con un piglio tra l'ironico e il divertito, esse svelano gli artifici del discorso amoroso, rendendo quasi necessaria una contro-replica che ristabilisca gli equilibri.

Nella sequenza prescelta a titolo di esempio Silvandre manifesta la sua sofferenza per la lontananza di Clorinde, costretta a partire per dovere di obbedienza nei confronti della madre:

Il y a si longtemps que ie vis, ou plustot que ie meurs en l'attente de vous revoir, belle et chaste Clorinde, que ie recognois par le ressentiment, qu'il n'est point de douleur égalle à celle que vostre absence me cause (p. 368).

\section{Risponde Clorinde:}

Ie veux croire, discret Silvandre, que si vous m'aymez passionnément, comme vous dites, vous pouvez ressentir quelque sorte de déplaisir en mon absence, mais non pas si cuisant que vous me le figurez. Les Amans ont cette mauvaise coustume de se dire tousiours mourants, et reduits à l'extremité, au moindre sentiment de leur passion [...] Il me semble que ie vous ay oüy dire autres fois, que si vous estiez assuré que ie vous voulusse du bien, vous seriez le plus content homme du monde. Or ie vous asseure, pour vostre satisfaction, que non seulement ie vous veux du bien, mais qu'encore ie vous ayme chastement [...] (pp. 371-372).

La replica di Silvandre non lascia dubbi sulla sua cieca obbedienza all'amata. Così infatti egli conclude questo scambio: «Ie desadvouë, ma belle Clorinde, toutes les plaintes que ie vous ay iamais faites».

Applicato a molti dei luoghi comuni dell'espressione amorosa (lontananza, dono di un ritratto, gelosia), questo meccanismo di smascheramento del cliché espressivo finalizzato a modificare l'atteggiamento dell'interlocutore, si ripete e si diversifica, creando molteplici spunti narrativi e imperniando lo scambio epistolare su una serrata alternanza di lettere e repliche che riproduce i ritmi e le sottigliezze della conversazione mondana.

La seconda esemplificazione è ricavata da un altro segretario galante contemporaneo, l'anonimo Secretaire des secretaires pubblicato per la prima volta a Rouen, per i tipi di M. Gorgeu, nel 161937. Dopo un Advertissement pour dicter les Lettres

(37) Le Secretaire des secretaires, ou le thresor de la plume françoise, contenant la manière de composer et dicter toutes sortes de lettres missives, avec quelques lettres facetieuses. L'edizione consultata è quella rivista e aumentata «d'une grande quantité de lettres» (Rouen, J. Caillové, 1624). 
Missives, seguito da una sezione contenente un elenco di formule di apertura, di raccordo e di chiusura adattabili a qualsiasi tipo di composizione epistolare, inizia la raccolta vera e propria formata da lettere non numerate, introdotte da un titolo e appartenenti a diverse tipologie. Frammista ad alcune coppie di lettere, o biglietti, e relative risposte spicca una breve corrispondenza composta da cinque missive che narrano la storia di Floridon e Calisthé $e^{38}$. Rispetto ad altre analoghe sequenze, questa si contraddistingue per una più evidente impostazione narrativa. Il progredire della vicenda è scandito dal breve, ma intenso alternarsi delle lettere dei due amanti, che si saldano strettamente l'una con l'altra fornendo le coordinate essenziali al dipanarsi della storia. Nella prima si assiste infatti al nascere della passione, che Floridon teme possa essere ostacolata dal timore di Calisthée nei confronti del marito. La risposta che segue fuga ogni timore e dà voce all'amore trionfante per poi lasciare posto all'angoscia causata dalla scoperta della relazione d'amore da parte del marito e infine alle rassicurazioni reciproche:

Les plaisirs sont de peu de durée, cher Floridon, leurs beaux jours s'eclipsent tout aussi tost. On ne se peut permettre une longue iouyssance puisqu'elle vient des mains de la fortune. C'est cette inconstante qui nous a donné un tel coup de sa legereté, que mon mary soit adverty de nos amours. Ie le vous escrits à mon grand regret, ces caracteres en portent le deuil [...] [N]'estoit que la ioye suit la tristesse, comme l'Esté l'Hyver, i'aurois desià franchy le pas de ceste vie, pour m'affranchir de mes peines. Mais le temps un iour nous donnera l'heure en laquelle nous nous vengerons en recevant l'usure de nostre patience [...] (Calisthée escrit à Floridon, pp. 486-487)

[...] mais si fut-il que i'ouvre la porte aux douleurs, puis que vostre lettre m'est un article de foy, et que vous souffrez mille gehennes pour m'avoir aimé. Ie ne doute point que l'envie n'aye bourdonné aux oreilles de mes parents quelque chose de nos amours, et que la fortune ialouse de nostre contentement, ne nous aye fait ce lasche trait: mais vous avez l'ame si belle et genereuse, que vous repousserez constamment toutes ces attaques, pour honorer tousjours de vostre amour vostre fidelle Floridon. (Response de Floridon, p. 489)

Intorno ai due nodi lettera-risposta si dispone questa semplice costruzione narrativa il cui sviluppo nasce dall'interazione di due movimenti opposti, l'uno euforico e coincidente con la prima coppia di missive, l'altro disforico e coincidente con la seconda. Ogni risposta infatti si allinea, sul piano del contenuto, con la lettera che la precede, confermandone e rafforzandone gli intenti e accrescendo conseguentemente lo spessore della storia. Le risposte assolvono dunque in questo caso il compito di rinsaldare i binari entro i quali si dispone e trova sviluppo la materia narrata.

Per illustrare l'ultima tipologia di replica epistolare ricorreremo ad un altro testo seicentesco: le Lettres meslées di Tristan L'Hermite, pubblicate da Augustin Courbé nel 1624 e comprendenti varie sezioni tra cui una di Lettres amoureuse. ${ }^{39}$ Ennesima applicazione di tematiche e formule espressive codificate dalla letteratura sentimentale dell'epoca, esse non nascondono la vicinanza e la consonanza con la produzione poetica amorosa del loro autore. Costruite su un asse comunicativo univoco, dall'amante all'amata, non presentano particolari elementi unificanti. Rivolte

(38) Le lettere non sono disposte in una serie continua, ma alternate con altre non attinenti. Complessivamente occupano le pagine 483-490.

(39) L'edizione di riferimento è la seguente: F. Tristan L'Hermite Lettres meslées. Edition critique par C. Grisé. Genève-Paris, Droz-Minard, 1972. Il volume comprende 5 sezioni: Epistres dédicatoires, Lettres de consolation, Lettres amoureuses, Lettres béroïques, Lettres meslées. 
a destinatarie molteplici e spesso dotate di nome, tendono a condensarsi in serie di lunghezza disuguale che evidenziano al loro interno solo esili fili narrativi. Non sono presenti lettere di risposta. Compaiono tuttavia, seppur raramente, riferimenti a missive non rese note, ma che fungono da antecedente narrativo sul quale si agganciano le lettere a disposizione del lettore, assimilabili in qualche modo a delle risposte che garantiscono una certa progressione degli eventi evocati.

Questa presenza implicita e mascherata della risposta si manifesta in una breve sequenza di tre lettere rivolte dallo scrivente «à sa soeur d'alliance» che, incurante dei consigli dissuasivi del suo interlocutore, allaccia una relazione con O.O.O., personaggio di cattiva reputazione. Dopo averla compianta per le sue pene d'amore, il firmatario delle lettere le rimprovera di non aver seguito i suoi consigli e le rinfaccia la sua ingratitudine. Questo semplice schema narrativo articolato in tre fasi trae origine in una lettera della donna a sua volta scritta in risposta a una precedente missiva. Né l'una né l'altra sono direttamente leggibili, ma vengono evocate con precisione nella prima lettera della serie, che commenta e riprende i suoi antecedenti epistolari:

Ma soeur, vous estes devenue une grande rethoricienne depuis que vous lisez V.V.V., mais j'avoue que j'ay treuvé vostre responce plus eloquente que raisonnable. Vous avez suivy la metaphore dont je vous ay dépeint le danger où vous vous mettez en soufrant les visites de O.O.O., et pour ce que je vous ay mandé que ce commerce estoit une mer où vostre reputation couroit fortune de naufrage, vous m'écrivez que vous prevoyez bien ce danger et que vous estes marrie d'estre embarquée. Hélas! ma mignonne, s'il est ainsi que vous redoutiez tant soit peu le peril, et que mes conseils vous semblent utils, l'ancre n'est pas encore levée, le vent n'a pas enflé les voiles, vous estes encore dans le port, vous n'avez qu'à mettre pié à terre (XXVIII, A sa soeur d'alliance).

La dimensione dialogica emerge fin dalle prime righe della lettera XXVIII, che assume in questo modo il valore di una replica. E interessante notare come i legami con le lettere precedenti siano intessuti anche a livello formale, lungo il filo conduttore di una metafora filée che avvicina stilisticamente il discorso esplicito ai suoi presupposti impliciti.

Numerosi e diversificati sono gli esempi di risposte epistolari concepite come elementi aggreganti di nuclei di lettere a coloritura romanzesca. In un'indagine che è ben lontana dal proporsi come esaustiva, le campionature esaminate non intendono esaurire la varietà e in taluni casi la ricchezza di materiali epistolari che, pur concepiti come repertori di modelli spesso scarsamente originali e comunque sempre lontani dalle realizzazioni di pura finzione, presentano a volte, quasi allo stato embrionale, delle potenzialità narrative suscettibili di sviluppo. All'interno di questa tipologia di testi lo studio di alcune tra le espressioni più significative di questa deriva finzionale fa risaltare il ruolo non secondario della lettera di risposta, punto di congiunzione tra segmenti epistolari adiacenti. La sua funzione di raccordo fa sì che essa possa garantire un'aggregazione dei singoli elementi narrativi veicolati dalle lettere, orientando il contenuto delle stesse in una prospettiva evolutiva coincidente con una storia. Facendo emergere le affinità tra dinamiche dialogiche e dinamiche epistolari la risposta, da matrice minima di finzione quale essa appare nella produzione di manuali e formulari cinque-seicenteschi, tende ad espandersi nel corso del XVII secolo e ad accrescere le sue potenzialità agglomeranti fino a ritmare costruzioni narrative compiute, garantendo così il passaggio da forme episodiche di emergenza della finzione a composizioni romanzesche dotate di una sempre maggiore autonomia. Basti pensare alle Lettres de Babet, serrato duetto la cui architettura narrativa si articola sulla dialettica tra lettera e replica, esito significativo del percorso che conduce dall'insieme spesso indistinto dei florilegi epistolari, a cui neanche il romanzo di Boursault risulta del resto del tutto estraneo, alla forma letteraria compiuta del roman par lettres. 